\title{
Effects of acute hyperglycaemia on anorectal motor and sensory function in diabetes mellitus
}

\author{
A. Russo, R. Botten, M.-F. Kong*, I. M. Chapman, R. J. L. Fraser, M. Horowitz and W.-M. Sunt
}

Department of Medicine, University of Adelaide, Royal Adelaide Hospital, Adelaide, South Australia, *Department of Diabetes \& Endocrinology, Leicester General Hospital, Leicester, UK, and †Department of Internal Medicine, Division of Gastroenterology, University of Michigan, Ann Arbor, MI, USA

Accepted 17 June 2003

\begin{abstract}
Aims To determine the effects of acute hyperglycaemia on anorectal motor and sensory function in patients with diabetes mellitus.

Methods In eight patients with Type 1, and 10 patients with Type 2 diabetes anorectal motility and sensation were evaluated on separate days while the blood glucose concentration was stabilized at either $5 \mathrm{mmol} / \mathrm{l}$ or $12 \mathrm{mmol} / \mathrm{l}$ using a glucose clamp technique. Eight healthy subjects were studied under euglycaemic conditions. Anorectal motor and sensory function was evaluated using a sleeve/sidehole catheter, incorporating a barostat bag.

Results In diabetic subjects hyperglycaemia was associated with reductions in maximal $(P<0.05)$ and plateau $(P<0.05)$ anal squeeze pressures and the rectal pressure/volume relationship (compliance) during barostat distension $(P<0.01)$. Hyperglycaemia had no effect on the perception of rectal distension. Apart from a reduction in rectal compliance $(P<0.01)$ and a trend $(P=0.06)$ for an increased number of spontaneous anal sphincter relaxations, there were no differences between the patients studied during euglycaemia when compared with healthy subjects.
\end{abstract}

Conclusions In patients with diabetes, acute hyperglycaemia inhibits external anal sphincter function and decreases rectal compliance, potentially increasing the risk of faecal incontinence.

Diabet. Med. 21, 176-182 (2004)

Keywords anorectal motility, hyperglycaemia, faecal incontinence

\section{Introduction}

Anorectal dysfunction, leading to faecal incontinence, has been reported in up to $20 \%$ of unselected out-patients with diabetes and represents a substantial morbidity [1]. In such patients multiple anorectal motor and sensory dysfunctions have been documented; basal and squeeze pressures may be reduced, the internal anal sphincter is frequently unstable, and rectal sensation may be impaired [1-5].

Disordered anorectal function in diabetes has been attributed to irreversible autonomic neuropathy $[1,4,5]$; however, acute changes in blood glucose concentration have a substantial, reversible effect, on gastrointestinal motor and sensory

Correspondence to: Professor Michael Horowitz, Department of Medicine, Royal Adelaide Hospital, North Terrace, Adelaide, SA, 5000, Australia. E-mail: michael.horowitz@adelaide.edu.au function, in the oesophagus [6], stomach [7-10], small intestine [11], gallbladder [12], colon [13] and anorectum [14-16]. While most studies have evaluated normal subjects $[6,9,10,13-15]$, acute changes in blood glucose also affect gastrointestinal motility in diabetes $[8,10,17]$. Previous studies of anorectal motility in patients with diabetes have substantial limitations. In particular, the techniques used were often suboptimal, and only isolated aspects of anorectal function were evaluated, and perhaps most importantly $[1-4,18,19]$, no account was taken of the potential impact of blood glucose concentration on anorectal function. It is likely that some of the abnormalities observed in these studies reflect hyperglycaemia, rather than diabetes per se [14-16]. This could also account, at least in part, for the substantial discrepancies in previous studies $[2-4,18,19]$. The effects of hyperglycaemia on anorectal motility have hitherto been evaluated only in normal subjects [14-16]. 
The aim of this study was to determine the effects of hyperglycaemia on anorectal motor and sensory function in a heterogeneous group of patients with Type 1 and Type 2 diabetes.

\section{Subjects and methods}

\section{Subjects}

Eight Type 1 [two female, six male; mean age $49 \pm 2$ years; body mass index (BMI) $29 \pm 1 \mathrm{~kg} / \mathrm{m}^{2}$ ], 10 Type 2 (two female, eight male; age $54 \pm 3$ years; BMI $33 \pm 1 \mathrm{~kg} / \mathrm{m}^{2} ; P<0.05$ vs. controls) patients with diabetes mellitus, and eight healthy male subjects (age $58 \pm 3$ years; BMI $28 \pm 3.6 \mathrm{~kg} / \mathrm{m}^{2}$ ) were studied. The duration of known diabetes was $24 \pm 5$ years in Type 1 patients and $7 \pm 1$ years in Type 2 patients; glycated haemoglobin was $8.1 \pm 0.5 \%$ (normal range $4-6 \%$ ). All Type 1 patients were receiving insulin at least twice a day; four Type 2 patients were taking oral hypoglycaemic medication (metformin and gliclazide) and two were treated with insulin; the remaining four Type 2 patients were treated by diet. Patients with diabetes were recruited by one of the investigators (M.-F.K.) by random numbers from out-patients attending the Royal Adelaide Hospital and not on the basis of gastrointestinal symptoms. None of the patients was taking medication known to affect gastrointestinal function or had previous gastrointestinal surgery, apart from uncomplicated appendectomy. Three of the four female patients had children; there was no history of significant obstetric trauma. None of the normal subjects had a history of gastrointestinal or urinary symptoms, previous gastrointestinal surgery or was taking medication known to affect gastrointestinal function. Written, informed consent was obtained from each subject and the study protocol was approved by the Human Ethics Committee of the Royal Adelaide Hospital.

\section{Protocol}

In each subject with diabetes measurements of anorectal motility and sensation were performed on two separate days, in single-blind fashion, while the blood glucose concentration was maintained at either $5 \mathrm{mmol} / \mathrm{l}$ ('euglycaemia') or $12 \mathrm{mmol} / \mathrm{l}$, using a glucose clamp technique [15]. The order of the two studies was randomized and separated by approximately 1 week. In the normal subjects measurements of anorectal motility and sensation were performed once, during euglycaemia.

On each study day subjects fasted from approximately $24.00 \mathrm{~h}$ (the Type 1 patients modified their morning dose of insulin) and did not undergo any bowel preparation, but were encouraged to empty their bowels before arriving at the laboratory at $09.00 \mathrm{~h}$. Each subject then completed a questionnaire assessing lower gastrointestinal symptoms [20]. The frequency and severity of faecal incontinence and urgency were quantified as: $0=<1 /$ week, $1=\geq 1$ but $<3 /$ week, $2=\geq 3$ but $<7 /$ week, $3=$ each day. Digital examination was then performed to ensure that the rectum was empty. Intravenous lines were placed in antecubital veins of both arms, one for intravenous infusion of insulin, saline and glucose, and the other for blood sampling. The latter arm was kept heated, using an electric pad, to 'arterialize' the venous blood. Measurements of anorectal motility and sensation were performed after the blood glucose concentration had been stabilized at the desired level for at least $60 \mathrm{~min} \mathrm{[15];} \mathrm{immediately} \mathrm{following} \mathrm{this,} \mathrm{on}$ the study performed during euglycaemia, each diabetic patient underwent an assessment of autonomic nerve function [21]. The intravenous infusion was then stopped and the patient given a meal before leaving the laboratory.

\section{Stabilization of blood glucose concentrations}

After a baseline blood glucose measurement, infusions of $25 \%$ glucose, normal saline and insulin (Actrapid; Novo Nordisk Pharmaceuticals, NSW, Australia) were commenced. A 30-60min 'run-in' period was used to achieve the desired blood glucose concentration, followed by a 60-min 'stabilization' period. At this time a bolus of either saline or glucose was administered over $10 \mathrm{~min}$; the volume of the bolus was the same on both days. The insulin infusion rate was initially variable, based on the blood glucose concentrations, followed by $60 \mathrm{mU} / \mathrm{kg}$ per hour for $30 \mathrm{~min}$ and $22.5 \mathrm{mU} / \mathrm{kg}$ per hour thereafter. The saline infusion was maintained at $50 \mathrm{ml} / \mathrm{h}$ on both days, while the $25 \%$ glucose infusion was variable. Venous blood samples were taken every $5 \mathrm{~min}$ throughout the run-in and stabilization periods and every 10-15 min thereafter, and glucose measured using a portable glucose meter (Medisense Companion II glucometer; Medisense Inc, Waltman, MA, USA).

\section{Measurement of anorectal motility and sensation}

The subject lay in the left lateral position, with the hips flexed at $90^{\circ}$, for the duration of the measurements. The manometric assembly (outer diameter $4.4 \mathrm{~mm}$ ) incorporated five sideholes, a sleeve sensor and a $5.5-\mathrm{cm}$ highly compliant polyethylene bag $(250 \mathrm{ml})$. The sleeve $(3 \mathrm{~cm}$ long), and three sideholes separated by $1 \mathrm{~cm}$, were positioned in the anal canal [15]. The lower and upper rectal sideholes $(5$ and $16 \mathrm{~cm}$, respectively, from the anal verge) were separated by the bag (located between 6.5 and $12 \mathrm{~cm}$ from the anal verge). A 'balloon' measurement port at $8.5 \mathrm{~cm}$ was attached to a barostat (Distender Series II; G\&J Electronics, Ontario, Canada) and used to measure intrabag pressure or volume, as appropriate. A series of trial distensions at the desired volumes revealed that the bag generated no pressure until $250 \mathrm{ml}$. All channels, except the 'balloon' channel, were perfused with degassed, distilled water at a rate of $0.15 \mathrm{ml} / \mathrm{min}$ by a pneumohydraulic pump.

Anorectal pressures were initially recorded for a 20-min 'baseline' period under resting conditions. After this time the subject was asked to squeeze the anal sphincter muscles maximally three times for $15 \mathrm{~s}$, with a 1-min 'rest' in between. Following a 10-min 'rest', rectal distensions were commencedthree different distensions were performed in randomized order, each separated by $10 \mathrm{~min}$. These included: (i) an intermittent manual distension utilizing a hand-held syringe, with incremental volumes of 10, 20, 40, 60, 100, 150 and $200 \mathrm{ml}$ of air; (ii) manual distension using identical volumes, but in random order; and (iii) an isobaric (barostat) distension using increments of $1 \mathrm{mmHg}$, from 4 to $20 \mathrm{mmHg}$ above the minimal rectal pressure (MRP). Each inflation was maintained for $1 \mathrm{~min}$ and the distension protocol was terminated at a volume of $200 \mathrm{ml}$, 
pressure of $20 \mathrm{mmHg}$, or if the subject experienced discomfort or pain. A 1-min 'rest' was allowed between each isovolumetric distension. During rectal distensions subjects were asked to report verbally the nature of the rectal sensation by assigning a score at the appropriate times: $0=$ no sensation, $1=$ possible sensation, 2 = slight feeling of distension, 3 = definite sensation of distension, $4=$ mild desire to pass wind, $5=$ desire to pass wind, $6=$ presence of a stool, $7=$ mild desire to defecate, $8=$ desire to defecate, $9=$ discomfort but not pain, $10=$ pain. Five minutes after the completion of all of the distensions, subjects were asked to increase their intra-abdominal pressure by forced expiration against a resistance; blowing up a $25-\mathrm{cm}$ party balloon for $15 \mathrm{~s}$ (Ansell International, Glen Waverley, Victoria, Australia).

Data were recorded using custom-written software (DAD, written by Assoc. Prof. G. S. Hebbard using Labview, National Instruments Corp., Austin, TX, USA) on a computer (Powermac 7100; Apple, Cupertino, CA, USA).

\section{Analysis of anorectal motility}

Data were analysed 'blindly' with the aid of Acqknowledge program (Acqknowledge; Biopac Systems, Santa Barbara, CA, USA).

\section{Spontaneous anal relaxations}

The number, duration and depth of spontaneous anal relaxations were calculated for the 20 -min baseline period (60 min after the establishment of the desired blood glucose concentration) [22].

\section{Anorectal pressures}

The following parameters were evaluated: (i) mean basal pressure, defined as the average pressure at the end of respiration sustained for at least 2 min during the 20-min baseline period; (ii) mean squeeze maximum and plateau pressure, defined as the visual mean of the pressure profile; and (iii) the mean pressure achieved when the subject was blowing up a party balloon $[15,23]$.

\section{Rectal pressure-volume relationships}

The mean pressure (or volume for the isobaric distension) generated in the bag at each of the distending volumes or pressures was recorded. For the isobaric distension only data from, and including, $12 \mathrm{mmHg}$ were evaluated [23,24]. The MRP was defined as the minimum pressure which induced continuous fluctuations in the volume tracing [23].

\section{Recto-anal inhibitory reflex (RAIR)}

This anal reflex was scored when $80 \%$ of maximal relaxation was achieved, and analysed for onset, duration and depth $[25,26]$.

\section{Perception}

The threshold volume for perception of rectal distension and the volumes at which definite sensations of distension, presence of a stool, and mild desire to defecate were recorded [18,26].

\section{Evaluation of autonomic nerve function}

Parasympathetic function was assessed by evaluation of the heart rate during deep breathing ( $\mathrm{R}-\mathrm{R}$ interval) and the heart rate response to standing ('30:15' ratio); sympathetic function was assessed by the change in systolic blood pressure on standing. Each test was scored as 0 (normal), 1 (borderline), or 2 (abnormal) and a total score of $\geq 3$ was taken as evidence of definite autonomic neuropathy [21].

\section{Statistical analysis}

Baseline, squeeze, and spontaneous anal relaxations were analysed using the non-parametric Mann-Whitney $U$ - or Wilcoxon rank sum test, as appropriate. Intrabag pressures or volumes and sensation scores were compared between and within groups using analysis of variance for repeated measures (ANOVA; Statview 5, SAS Institute Inc., Cary, NC, USA). In the rectal distension protocols data at maximum distension (pressure or volume) were evaluated a priori, as these were likely to be most sensitive in detecting differences. The number of patients with diabetes that was studied $(n=18)$ was based on power calculations derived from our previous study which evaluated the effects of hyperglycaemia on anorectal function in eight normal subjects [15]. As there were no differences in parameters of anorectal motility between Type 1 and Type 2 patients, these data were pooled. Data are presented as mean (SEM). A Pvalue of $<0.05$ was considered to be significant.

\section{Results}

The study protocol was well tolerated and no adverse events were reported during either euglycaemia or hyperglycaemia. Two patients (one Type 1 and one Type 2) reported faecal incontinence and five urgency (two Type 1 and three Type 2). There was no significant difference in the number of bowel actions per week between diabetic subjects and normals ( $9 \pm 4$ /week vs. 7 \pm 3 /week). Eight of 17 subjects had definite autonomic neuropathy; in one patient autonomic nerve function was not evaluated because of technical difficulties. There was no significant difference in parameters of anorectal motility in those with autonomic neuropathy compared with the remainder of the group (data not shown). Complete data for the rectal distension protocols were not obtained in all cases because of technical difficulties, including subject intolerance to high volumes and bag rupture or over-pressurization during isobaric distensions.

Mean blood glucose concentrations closely approximated the desired range $(11.7 \pm 0.2 \mathrm{mmol} / \mathrm{l}$ during hyperglycaemia and $5.1 \pm 0.2 \mathrm{mmol} / \mathrm{l}$ during euglycaemia).

\section{Spontaneous anal relaxations}

During the 20-min baseline period there was no difference in the number, duration or depth of spontaneous anal relaxations between hyperglycaemia and euglycaemia in the diabetic subjects. There was a trend for the number of spontaneous anal relaxations to be greater in the patients during euglycaemia when compared with controls $(2.0 \pm 0.6$ vs. $0.3 \pm 0.3, P=0.06)$.

\section{Anorectal pressures}

Both the maximum squeeze pressure $(P<0.05)$ and the squeeze plateau pressure $(P<0.05)$, were less at a blood glucose 
Table 1 Parameters of anorectal motility in patients with diabetes studied at blood glucose concentrations of 5 and $12 \mathrm{mmol} / \mathrm{l}$ $(n=18)$, and normal subjects $(n=8)$ studied during euglycaemia (mean \pm SEM)

\begin{tabular}{llll}
\hline & \multicolumn{2}{c}{ Diabetes } & Normal \\
\cline { 2 - 4 } Parameter & $5 \mathrm{mmol} / 1$ & $12 \mathrm{mmol} / \mathrm{l}$ & \\
\hline Spontaneous anal relaxations & & & \\
$\quad$ Number & $2(0.6)$ & $1.4(0.5)$ & $0.3(0.3)$ \\
$\quad$ Depth (mmHg) & $42(10)$ & $28(3)$ & $15(0)$ \\
$\quad$ Duration (s) & $28(5)$ & $22(3)$ & $15(0)$ \\
Minimum basal pressure (mmHg) & $50(5)$ & $47(4)$ & $41(4)$ \\
Squeeze maximum pressure $(\mathrm{mmHg})$ & $159(13)$ & $141(10)^{*}$ & $164(27)$ \\
Squeeze plateau pressure (mmHg) & $114(12)$ & $94(8)^{*}$ & $118(14)$ \\
Anal pressure while blowing up a balloon $(\mathrm{mmHg})$ & $85(10)$ & $83(11)$ & $113(21)$ \\
\hline
\end{tabular}

* $P<0.0512$ vs. $5 \mathrm{mmol} / \mathrm{l}$ for diabetes group.
Table 2 The volume required to induce internal anal sphincter (IAS) relaxation in response to rectal balloon distension in patients with diabetes studied at blood glucose concentrations of 5 and $12 \mathrm{mmol} / \mathrm{l}$ $(n=18)$ and normal subjects $(n=8)$ during three distension protocols (mean \pm SEM)

\begin{tabular}{|c|c|c|c|}
\hline \multirow[b]{2}{*}{ Type of distension } & \multicolumn{2}{|c|}{ Diabetes } & \multirow[t]{2}{*}{ Normal } \\
\hline & $5 \mathrm{mmol} / \mathrm{l}$ & $12 \mathrm{mmol} / \mathrm{l}$ & \\
\hline \multicolumn{4}{|l|}{ Manual } \\
\hline First relaxation $(\mathrm{ml})$ & $39(7)$ & $42(11)$ & $26(4)$ \\
\hline Sustained relaxation $(\mathrm{ml})$ & $197(16)$ & $175(19)$ & $175(19)$ \\
\hline \multicolumn{4}{|l|}{ Random manual } \\
\hline First relaxation (ml) & $98(24)$ & $63(14)$ & $40(5)$ \\
\hline Sustained relaxation $(\mathrm{ml})$ & $200(21)$ & $194(21)$ & $168(16)$ \\
\hline \multicolumn{4}{|l|}{ Isobaric } \\
\hline First relaxation $(\mathrm{mmHg})$ & $9(1)$ & $10(1)$ & $7(1)$ \\
\hline Sustained relaxation $(\mathrm{mmHg})$ & $13(1)$ & $14(1)$ & $11(2)$ \\
\hline
\end{tabular}

concentration of $12 \mathrm{mmol} / \mathrm{l}$ when compared with $5 \mathrm{mmol} / \mathrm{l}$ (Table 1). Anal pressures were not significantly different between the patients during euglycaemia and controls.

\section{Recto-anal inhibitory reflex}

In the diabetic subjects there was no effect of blood glucose concentration on the volumes at which the first and sustained internal anal sphincter (IAS) relaxation occurred during any of the three distension protocols; there was also no difference between the patient and normal groups (Table 2). There were also no differences in either the depth or duration of the RAIR between hyperglycaemia and euglycaemia, or between the diabetics and normals during manual or randomized manual distension (data not shown).

\section{Pressure-volume relationship}

\section{Incremental manual distension}

Intrabag pressure increased with increasing rectal distending volume $(P<0.01)$. In the patients there was no difference in intrabag pressure at any volume between hyperglycaemia and euglycaemia, e.g. at a volume of $200 \mathrm{ml}: 31.4 \pm 2.5 \mathrm{mmHg}$ vs. $32.9 \pm 3.1 \mathrm{mmHg}$. There was a trend $(P=0.07)$ for intrabag pressure to be greater in the diabetic subjects during euglycaemia when compared with controls; at a volume of $200 \mathrm{ml}$ this difference was significant $(32.7 \pm 2.7 \mathrm{mmHg}$ vs. $25.8 \pm 4.3 \mathrm{mmHg}, P<0.01)$.

\section{Random manual distension}

There was no difference between hyperglycaemia and euglycaemia in intrabag pressure in the patients, e.g. at a volume of $200 \mathrm{ml}: 33.6 \pm 3.7 \mathrm{mmHg}$ vs. $31.8 \pm 3.6 \mathrm{mmHg}$. Intrabag pressure was higher in the patients during euglycaemia than in controls at a volume of $200 \mathrm{ml}(33.4 \pm 3.4 \mathrm{mmHg}$ vs. $27.2 \pm$ $4.1 \mathrm{mmHg}, P<0.05)$.

\section{Isobaric distension (Fig. 1)}

MRP was $9.0 \pm 0.6 \mathrm{mmHg}$ during euglycaemia and $10.0 \pm$ $0.6 \mathrm{mmHg}$ during hyperglycaemia in patients and $9.0 \pm$ $0.9 \mathrm{mmHg}$ in controls. MRP was no different between patients and controls, nor affected by blood glucose concentration. Intrabag volume increased with increasing pressure $(P<0.01)$. Intrabag volume was less during hyperglycaemia compared with euglycaemia at each of the distending pressures $(P=0.06)$ and this difference was significant at a pressure of $20 \mathrm{mmHg}(124.0 \pm 23.6 \mathrm{ml}$ vs. $150.5 \pm 18.2 \mathrm{ml} ; P<0.05)$. In the patients intrabag volume during euglycaemia tended to be less at each of the distending pressures when compared with controls $(P=0.07)$, with a significant difference at a pressure of $20 \mathrm{mmHg}(148.7 \pm 16.4 \mathrm{ml}$ vs. $179.3 \pm 19.0 \mathrm{ml} ; P<0.01)$.

\section{Sensation}

Rectal balloon distension was perceived by all subjects.

\section{Manual distension}

In the diabetic subjects mean scores for perception of rectal sensation were slightly higher during hyperglycaemia compared with euglycaemia, but this difference was not significant, e.g. at a volume of $200 \mathrm{ml}: 7.2 \pm 0.5$ vs. $6.4 \pm 0.5$ (NS). 

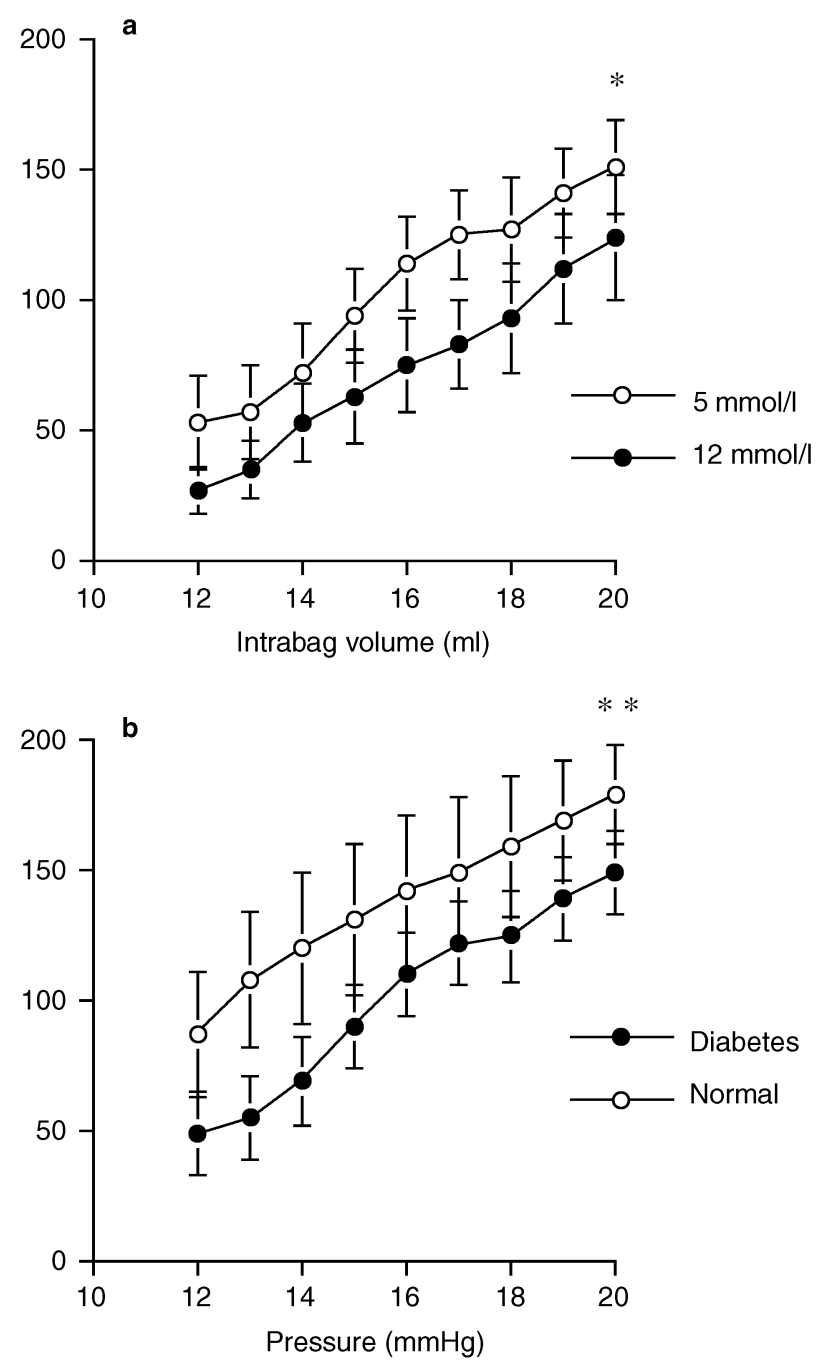

Figure 1 Intrabag volume $(\mathrm{ml})$ during isobaric rectal distension in (a) patients with diabetes during hyperglycaemia and euglycaemia $(n=12)$ and (b) patients with diabetes during euglycaemia $(n=14)$ and healthy subjects $(n=8)$. Data are mean \pm SEM. $* P<0.05 ; * P<0.01$.

There was no significant difference in perception of rectal sensation between patients and controls at any of the distending volumes (e.g. at a volume of $200 \mathrm{ml} ; 6.4 \pm 0.5$ vs. $6.4 \pm 0.6$, NS).

\section{Random manual distension}

There were no significant differences in sensation scores between the two blood glucose concentrations, nor between patients and controls (Table 3).

\section{Isobaric distension}

There were no significant differences in the perception of rectal sensation during isobaric distension at any of the distending pressures between the two blood glucose concentrations, or between patients and controls (Table 3 ).

There were no differences in threshold volumes for first perception or the threshold volume for perception of the bag, stool or desire to defecate between the two groups (i.e.
Table 3 The threshold volume required to induce (a) perception of rectal balloon distension, (b) presence of stool and (c) mild desire to defecate in patients with diabetes studied at blood glucose concentrations of 5 and $12 \mathrm{mmol} / \mathrm{l}$ and normal subjects during three distension protocols (mean $\pm \mathrm{SE})$

a.

\begin{tabular}{|c|c|c|c|}
\hline \multirow[b]{2}{*}{ Type of distension } & \multicolumn{2}{|c|}{ Diabetes } & \multirow[t]{2}{*}{ Normal } \\
\hline & $5 \mathrm{mmol} / \mathrm{l}$ & $12 \mathrm{mmol} / \mathrm{l}$ & \\
\hline Manual (ml) & $39(7)$ & $28(8)$ & $35(11)$ \\
\hline Random manual (ml) & $52(11)$ & $39(9)$ & $51(18)$ \\
\hline Isobaric $(\mathrm{mmHg})$ & $8(1)$ & $9(1)$ & $9(2)$ \\
\hline \multicolumn{4}{|l|}{ b. } \\
\hline & \multicolumn{2}{|c|}{ Diabetes } & Normal \\
\hline Type of distension & $5 \mathrm{mmol} / \mathrm{l}$ & $12 \mathrm{mmol} / \mathrm{l}$ & \\
\hline Manual (ml) & $128(11)$ & $110(10)$ & $133(28)$ \\
\hline Random manual (ml) & $125(14)$ & $141(14)$ & $163(13)$ \\
\hline Isobaric $(\mathrm{mmHg})$ & $12(2)$ & $16(1)$ & $12(2)$ \\
\hline \multicolumn{4}{|l|}{ c. } \\
\hline & \multicolumn{2}{|c|}{ Diabetes } & Normal \\
\hline Type of distension & $5 \mathrm{mmol} / \mathrm{l}$ & $12 \mathrm{mmol} / \mathrm{l}$ & \\
\hline Manual (ml) & $134(11)$ & $131(14)$ & $115(22)$ \\
\hline Random manual (ml) & $149(17)$ & $145(13)$ & $150(0)$ \\
\hline Isobaric $(\mathrm{mmHg})$ & $13(2)$ & $16(1)$ & $12(1)$ \\
\hline
\end{tabular}

hyperglycaemia vs. euglycaemia and patients vs. controls) for each of the distension protocols (Table 3 ).

\section{Discussion}

To our knowledge this study represents the first evaluation of the effects of hyperglycaemia on anorectal motility in patients with diabetes. Our observations establish that in this group acute hyperglycaemia affects external anal sphincter function, as well as rectal compliance. At a blood glucose of $12 \mathrm{mmol} / \mathrm{l}$, anal squeeze and plateau pressures and rectal compliance were reduced compared with euglycaemia. In contrast, the blood glucose concentration had no significant effect on the perception of rectal distension. These observations have implications for an understanding of the pathophysiology of anorectal dysfunction and faecal incontinence in diabetes.

The pathogenesis of disordered gastrointestinal motility in diabetes is now recognized to be multifactorial; those factors which appear to be dominant, autonomic neuropathy and glycaemic control, are closely related [10]. While the prevalence of disordered motility may be higher in patients with cardiovascular autonomic neuropathy than in those without, the association is relatively weak [10]. We intentionally studied a heterogeneous group of patients with long-standing diabetes; it should be recognized that our study was not designed 
primarily to evaluate the impact of diabetes per se (Type 1 or Type 2), or its microvascular complications, on anorectal function. Furthermore, the diabetic and control groups were not matched for gender or age. Hence, observations relating to this component of the study should be viewed circumspectly. There were also relatively few differences between diabetic subjects studied during euglycaemia and controls; in the patients there was a reduction in rectal compliance that was most evident during barostat distensions and a trend for an increased number of spontaneous internal anal sphincter relaxations. These observations are consistent with previous reports in non-diabetic patients with faecal incontinence $[22,25]$. We have reported an increased frequency of spontaneous internal anal sphincter relaxations in patients with diabetes who have faecal incontinence [18] (the non-significant trend for an increase in the current study may well represent a type 2 error); moreover, the occurrence of internal anal sphincter relaxations is associated with leakage, as residual anal pressure is lower than the rectal pressure and compensatory external anal sphincter contraction is impaired [22]. The reduction in rectal compliance would also favour incontinence.

We have reported in normal subjects that elevation of the blood glucose to $12 \mathrm{mmol} / \mathrm{l}$ is associated with an increased number of spontaneous anal relaxations, a reduction in squeeze pressures and enhanced rectal compliance and perception of rectal distension, when compared with euglycaemia [15]. Chey et al. have also evaluated the effects of hyperglycaemia (blood glucose approximately $15 \mathrm{mmol} / \mathrm{l}$ ) on anorectal function in normal subjects and reported that both the perception of rectal distension and the recto-anal inhibitory reflex were blunted during hyperglycaemia [14]. These authors did not observe any effect of hyperglycaemia on external or internal anal sphincter function. More recently, Avsar et al. reported, also in normal subjects, that the perception of 'ramplike' rectal distension was attenuated, whereas the perception of rapid, intermittent, rectal distension was not affected by hyperglycaemia [16]. These observations suggest that the effects of hyperglycaemia on the perception of rectal sensation and rectal compliance may be dependent on the methodology used.

Our study establishes that acute hyperglycaemia impairs the function of the external anal sphincter in patients with diabetes, as in healthy subjects [15]. In this latter study, hyperglycaemia increased rectal compliance, as assessed by intermittent incremental rectal balloon distension with a hand-held syringe [15]. In contrast, in the current study there was no difference in the rectal pressure-volume relationships between hyperglycaemia and euglycaemia in response to either incremental or random rectal balloon distension, but a reduction in compliance was evident during barostat distensions in the patients; the barostat technique is known to be safe and reproducible [24]. When compared with healthy subjects, rectal compliance was reduced in all three experimental conditions, indicative of a 'stiffer' rectum in those with diabetes. Such a non-compliant rectum may require more time to 'accommodate' rectal distension. Accordingly, while hyperglycaemia had no effect on the response to the faster manual distension, the slower barostat distension may have provided the 'time window' required to detect differences in accommodation between euglycaemia and hyperglycaemia.

Previous studies have demonstrated that hyperglycaemia affects the perception of rectal distension in healthy subjects [15] and suggest that diabetes may be associated with impaired rectal sensitivity $[2,3]$. The absence of any difference in perception of rectal distension between euglycaemia and hyperglycaemia, or between the two groups, may potentially be attributable to the slow inflation rate associated with the barostat distension. Scores for perception of rectal distension were also less during the barostat distensions when compared with manual distensions. We also employed a numerical system to quantify rectal sensation, in contrast to our previous approach of recording specific sensations, e.g. perception and desire to defecate $[15,18]$. It should also be recognized that random manual distension provides little additional information about rectal sensory and motor function, but was used to avoid the potential for patient response bias [26]. However, rectal wall tension may require more time to recover when a larger volume distension is administered first in a random distension protocol. Accordingly, it may be appropriate to allocate a 'rest period' of greater than $1 \mathrm{~min}$ between distensions. The effects of hyperglycaemia on perception of rectal sensation during hyperglycaemia may therefore also be dependent on the method of balloon distension $[19,27]$. The apparent lack of association between rectal compliance and sensation is not surprising. Although little is known about the underlying mechanisms, diabetes may affect both somatic [28] and visceral sensation [29]. Furthermore, central processing is also affected by hyperglycaemia [30]. This may account for the observation that the reduced rectal compliance or a 'stiff' rectum was not associated with increased sensation.

A number of potential mechanisms may mediate the effects of blood glucose concentration on gut motor and sensory function $[10,30,31]$. A direct effect on smooth muscle is unlikely [10]. Hyperglycaemia suppresses parasympathetic (vagal) tone in normal subjects [6]. The concept that the effects of hyperglycaemia are mediated, at least in part, by impaired vagal activity is also supported by animal studies [32], which have also demonstrated the presence of glucose-responsive neurones in the central nervous system [33]. Neurones responsive to glucose have also been identified in the rat small intestine [34] and, presumably, also exist in humans. Hyperinsulinaemia is unlikely to play a major role $[10,13,31]$.

While the observed effects of acute hyperglycaemia on anorectal motor function in diabetic patients appear to be relatively modest, it should be recognized that such changes have been reported in patients with diabetes who have faecal incontinence. Accordingly, it appears likely that some of the reported abnormalities are attributable to hyperglycaemia [2$4,17,18]$. Further studies are indicated to determine whether 
the effects of hyperglycaemia are related to the presence of other diabetic complications, particularly autonomic neuropathy. Our observations suggest that improving glycaemic control may be of benefit in the treatment of symptomatic anorectal dysfunction and provide a compelling case for studies of anorectal function in patients with diabetes to be performed during euglycaemia.

\section{Acknowledgement}

This study was supported by a grant from the National Health and Medical Research Council of Australia.

\section{References}

1 Feldman M, Schiller LR. Disorders of gastrointestinal motility associated with diabetes mellitus. Ann Intern Med 1983; 98: 378-384.

2 Schiller LR, Santa Ana CA, Schmulen AC, Hendler RS, Harford WV, Fordtran JS. Pathogenesis of faecal incontinence in diabetes mellitus -evidence for internal-anal-sphincter dysfunction. N Engl J Med 1982; 307: 1666-1671.

3 Wald A, Tunuguntla K. Anorectal sensorimotor dysfunction in faecal incontinence and diabetes mellitus-modification with biofeedback therapy. N Engl J Med 1984; 310: 1281-1287.

4 Rogers J, Levy DM, Henry MM, Misiewicz JJ. Pelvic floor neuropathy: a comparative study of diabetes mellitus and idiopathic faecal incontinence. Gut 1988; 29: 756-761.

5 Wald A. Colonic and anorectal motility testing in clinical practice. Am J Gastroenterol 1994; 89: 2109-2115.

6 De Boer S, Masclee A, Lam W, Lamers C. Effect of acute hyperglycaemia on oesophageal motility and lower oesophageal sphincter pressure in humans. Gastroenterology 1992; 103: 775-780.

7 Schvarcz E, Palmer M, Aman J, Horowitz M, Stridsberg M, Berne C. Changes in blood glucose within the physiological range affect gastric emptying in normal subjects and patients with insulin-dependent diabetes mellitus. Gastroenterology 1997; 113: 60-66.

8 Fraser R, Horowitz M, Maddox A, Harding P, Chatterton B, Dent J. Hyperglycaemia slows gastric emptying in type I diabetes mellitus. Diabetologia 1990; 33: 675-680.

9 Hebbard GS, Samsom M, Sun WM, Dent J, Horowitz M. Hyperglycaemia affects proximal gastric motor and sensory function during small intestinal triglyceride infusion. Am J Physiol 1996; 271: 68146819.

10 Rayner CK, Samsom M, Jones KL, Horowitz M. Relationships between upper gastrointestinal motor and sensory function with glycaemic control. Diabetes Care 2001; 24: 371-381 (review).

11 Russo A, Fraser R, Horowitz M. The effect of acute hyperglycaemia on small intestinal motility in normal subjects. Diabetologia 1996; 39: 984-989.

12 Gielkens HA, van Oostayen JA, Frolich M, Biemond I, Lamers CB, Masclee AA. Dose-dependent inhibition of postprandial gallbladder motility and plasma hormone secretion during acute hyperglycaemia. Scand J Gastroenterol 1998; 33: 1074-1079.

13 Sims M, Hasler W, Chey W, Kim M, Owyang C. Hyperglycaemia inhibits mechanoreceptor-mediated gastrocolonic responses and colonic peristaltic reflexes in healthy humans. Gastroenterology 1995; 108: 350-359.

14 Chey WD, Kim M, Hasler WI, Owyang C. Hyperglycaemia alters perception of rectal distension and blunts the rectoanal inhibitory reflex in healthy volunteers. Gastroenterology 1995; 108: 1700-1708.
15 Russo A, Sun WM, Sattawatthamromg Y, Fraser R, Horowitz M, Andrews JM et al. Acute hyperglycaemia affects anorectal motor and sensory function in normal subjects. Gut 1997; 41: 494-499.

16 Avsar E, Ersoz O, Karisik E, Erdogan Y, Bekiroglu N, Lawrance R et al. Hyperglycaemia-induced attenuation of rectal perception depends upon pattern of rectal balloon inflation. Dig Dis Sci 1997; 42: 2206-2212.

17 Rayner CK, Verhagen MAMT, Hebbard GS, DiMatteo AC, Doran SM, Horowitz M. Proximal gastric compliance and perception in type 1 diabetes mellitus-effects of hyperglycaemia. Am J Gastroenterol 2000; 95: 1175-1183.

18 Sun WM, Katsinelos P, Horowitz M, Read NW. Disturbances in anorectal function in patients with diabetes mellitus and faecal incontinence. Eur J Gastroenterol Hepatol 1996; 8: 1007-1012.

19 Caruana BJ, Wald A, Hinds JP, Eidelman BH. Anorectal sensory and motor function in neurogenic faecal incontinence. Gastroenterology 1991; 100: 465-470.

20 Pecatori M, Anastasio G, Bottini C, Mentasti A. New grading and scoring for anal incontinence. Evaluation of 335 patients. Dis Colon Rectum 1992; 35: 482-487.

21 Horowitz M, Maddox AF, Wishart JM, Harding PE, Chatterton BE, Shearman DJ. Relationships between oesophageal transit and solid and liquid gastric emptying in diabetes mellitus. Eur J Nucl Med 1991; 18: 229-234.

22 Sun WM, Read NW, Miner PB, Kerrigan DD, Donnelly TC. The role of transient internal sphincter relaxation in faecal incontinence? Int $J$ Colorect Dis 1990; 5: 31-36.

23 Yeoh E, Sun W, Russo A, Ibanez L, Horowitz M. A retrospective study of the effects of pelvic irradiation for gynaecological cancer on anorectal function. Int J Radiat Oncol Phys 1996; 35: 1003-1010.

24 Bharuca AE. Fecal incontinence. Gastroenterology 2003; 124: 1672-1685.

25 Sun WM, Donnelly TC, Read NW. Impaired internal anal sphincter in a subgroup of patients with idiopathic faecal incontinence. Gastroenterology 1989; 97: 130-135.

26 American Gastroenterological Association. Medical Position Statement on anorectal testing techniques. Gastroenterology 1999; 116: 732-760.

27 Sun WM, Read NW, Prior A, Daly JA, Cheah SK, Grundy D. Sensory and motor responses to rectal distension vary according to rate and pattern of balloon inflation. Gastroenterology 1990; 99: 1008-1015.

28 Maxton DG, Whorwell PJ. Functional bowel symptoms in diabetes -the role of autonomic neuropathy. Postgrad Med J 1991; 67: 991-993.

29 Aitchison M, Fisher BM, Carter K, McKee R, MacCuish AC, Finlay IG. Impaired anal sensation and early diabetic faecal incontinence. Diabet Med 1991; 8: 960-963.

30 Russo A, Smout AJPM, Kositchaiwat C, Rayner C, Sattawatthamrong Y, Semmler J et al. The effect of hyperglycaemia on cerebral potentials evoked by rapid rectal distension in healthy humans. Eur J Clin Invest 1999; 29: 512-518.

31 Kong MF, King P, MacDonald IA, Blackshaw PE, Horowitz M, Perkins AC et al. Euglycaemic hyperinsulinaemia does not affect gastric emptying in type I and type II diabetes mellitus. Diabetologia 1999; 42: 365-372.

32 Ishigachi T, Tada H, Nakagawa K, Yamamura T, Takahashi T. Hyperglycaemia impairs antropyloric coordination and delays gastric emptying in conscious rats. Auton Neurosci 2002; 95: 112-120.

33 Routh VH. Glucose-sensing neurons. Are they physiologically relevant? Physiol Behav 2002; 76: 403-413.

34 Liu M, Seino S, Kirchgessner AL. Identification and characterization of glucoresponsive neurons in the enteric nervous system. J Neurosci 1999; 19: 10305-10317. 